\title{
Plane Transverse MHD Flow through Porous Media
}

\author{
M. H. Hamdan ${ }^{*}$, Roberto Silva-Zea ${ }^{2}$, Romel Erazo-Bone ${ }^{3}$, Fidel Chuchuca-Aguilar ${ }^{3}$, \\ Kenny Escobar-Segovia ${ }^{4}$
}

${ }^{1}$ Department of Mathematics \& Statistics, University of New Brunswick, Saint John, Canada

${ }^{2}$ Hidroingeniería S.A., Head of Research and Development, Guayaquil, Ecuador

${ }^{3}$ Universidad Estatal Península de Santa Elena, Santa Elena, Ecuador

${ }^{4}$ Escuela Superior Politécnica del Litoral, Guayaquil, Ecuador

Email: `hamdan@unb.ca, roberto.silva.zea2@gmail.com,raerazo@upse.edu.ec,fchuchuca@upse.edu.ec, kescobar@espol.edu.ec

How to cite this paper: Hamdan, M.H., Silva-Zea, R., Erazo-Bone, R., ChuchucaAguilar, F. and Escobar-Segovia, K. (2020) Plane Transverse MHD Flow through Porous Media. Journal of Applied Mathematics and Physics, 8, 2115-2128.

https://doi.org/10.4236/jamp.2020.810158

Received: August 15, 2020

Accepted: October 17, 2020

Published: October 20, 2020

Copyright $\odot 2020$ by author(s) and Scientific Research Publishing Inc. This work is licensed under the Creative Commons Attribution International License (CC BY 4.0).

http://creativecommons.org/licenses/by/4.0/

\begin{abstract}
Plane, transverse MHD flow through a porous structure is considered in this work. Solution to the governing equations is obtained using an inverse method in which the streamfunction of the flow is considered linear in one of the space variables. Expressions for flow quantities are obtained for finitely conducting and infinitely conducting fluids.
\end{abstract}

\section{Keywords}

Porous Media, MHD Flow, Transverse Magnetic Field

\section{Introduction}

Labropulu [1], elegantly stated that the movement of a fluid (liquid or gas) through a magnetic field may induce an electric field, and consequently a current. The current then interacts with the magnetic field to produce a force that acts on the flowing fluid. It has long been recognized that these interactions, and magneto-hydro-dynamic (MHD) flows in general, occur in nature and in man-made media and devices [1], and find applications in both natural settings, such as in plasma dynamics and in astrophysics [2], and in engineering applications, such as in power generation [3] [4].

Importance of these and many other applications have motivated various researchers to propose methods of solutions that are either specialized or approximate to provide satisfactory solutions to the inherently complex governing equations that lack complete analytical solutions. Many methods have been pro- 
posed and implemented, and have been reported in the literature (cf. [5]-[10] and the references therein).

Occurrence of MHD flows in the earth interior and in other porous materials underscores the need for studies of MHD flows in porous media. These flows enjoy a spectrum of engineering applications that have been investigated over the past few decades (cf. [4] and the references therein). Equations governing MHD flow through porous media have been thoroughly and elegantly analyzed by Geindreau and Auriault [4], who also presented a literature review of both the theoretical work, and the experiments that have been conducted in field.

Of particular interest to the current work is plane transverse MHD flow through a porous structure, wherein the magnetic vector field is considered normal to the plane of the flow, shown in Figure 1.

This type of flow might be of interest in some applications including lubrication theory where the magnetic field might have an impact on the load carrying capacity in mechanisms that include porous lining [11] [12] [13]. In the analysis to follow, we consider the steady, incompressible MHD flow through a porous structure with constant permeability. We parallel closely the elegant analysis and approach provided by Labropulu [1], in her study of Riabouchinsky flows of ordinary and second grade MHD fluids in free space in that we state, define and formulate the problem in a similar manner and assume that streamfunction is linear in one of the space variables. However, we present a method of solution that is based on the inverse approach introduced in by Hamdan [14] and received success in the solution of flow problems in porous media [15]. We provide expressions for the streamfunction, velocity components, vorticity, pressure functions and magnetic field.

While we consider in this work fluid flow in unconfined domains, solutions might be of interest in lubrication theory with porous lining under the influence of an electromagnetic field.

\section{Governing Equations}

Steady, magneto-hydro-dynamic (MHD) flow of an incompressible fluid, through a porous sediment, is governed by the following equations:

$$
\begin{aligned}
& \nabla \cdot \boldsymbol{v}=0 \\
& \nabla \cdot \boldsymbol{H}=0
\end{aligned}
$$

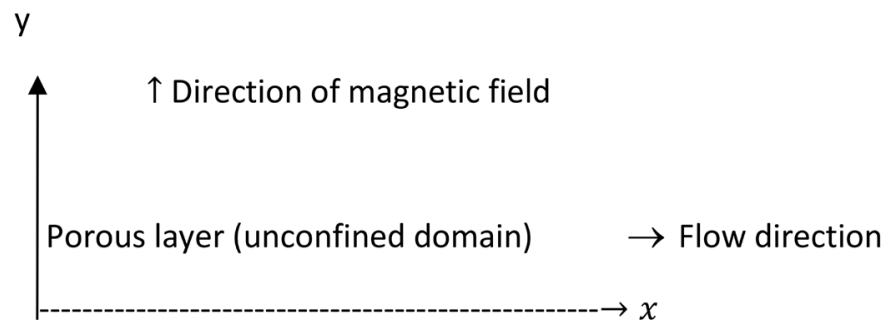

Figure 1. Representative sketch of flow direction. 


$$
\begin{gathered}
\rho(\boldsymbol{v} \cdot \nabla) \boldsymbol{v}=-\nabla p+\mu_{e} \nabla^{2} \boldsymbol{v}-\frac{\mu}{k} \boldsymbol{v}+\mu_{m}(\nabla \times \boldsymbol{H}) \times \boldsymbol{H} \\
\nabla \times(v \times \boldsymbol{H})-\frac{1}{\mu_{m} \sigma} \nabla \times \nabla \times \boldsymbol{H}=\mathbf{0}
\end{gathered}
$$

wherein $\mu_{m}$ is the magnetic permeability, $\boldsymbol{v}$ is the velocity vector field, $\boldsymbol{H}$ is the magnetic field, $p$ is the pressure function, $\rho$ is the fluid density, $\mu$ is the base fluid viscosity coefficient, $\mu_{e}$ is the effective viscosity of the fluid in the porous medium, $k$ is the (constant) permeability, $\sigma$ is the electrical conductivity, and $\nabla$ is the gradient operator. We point out that the electric current density is given by $\nabla \times \boldsymbol{H}$.

Employing the identity

$$
(\boldsymbol{v} \cdot \nabla) \boldsymbol{v}=\nabla\left[\frac{1}{2}|\boldsymbol{v}|^{2}-\boldsymbol{v} \times(\nabla \times \boldsymbol{v})\right]
$$

Equation (3) takes the form

$$
\rho \nabla\left[\frac{1}{2}|\boldsymbol{v}|^{2}-\boldsymbol{v} \times(\nabla \times \boldsymbol{v})\right]=-\nabla p+\mu_{e} \nabla^{2} \boldsymbol{v}-\frac{\mu}{k} \boldsymbol{v}+\mu_{m}(\nabla \times \boldsymbol{H}) \times \boldsymbol{H}
$$

Now, governing Equations (1), (4), and (6) represent a determinate system of seven scalar equations in the seven unknown functions $\boldsymbol{v}, \boldsymbol{H}$ and $p$, while Equation (2) represents an additional condition on $\boldsymbol{H}$ that signifies the absence of magnetic poles in the flow field.

Assuming the flow is in two space dimensions, $(x, y)$, then the velocity vector field is given by

$$
v=(u, v, 0)
$$

Assuming that that the magnetic field acts in a direction perpendicular to the plane of the flow, we take

$$
\boldsymbol{H}=(0,0, H)
$$

Equations (2) and (8) imply that $H_{z}=0$ and $H=H(x, y)$, where subscript notation denotes partial differentiation.

Using (7) and (8) in (1), (4) and (6), we obtain the following governing equations in components' form:

$$
\begin{gathered}
u_{x}+v_{y}=0 \\
\rho\left\{\frac{1}{2}\left[u^{2}+v^{2}\right]_{x}-v\left(v_{x}-u_{y}\right)\right\}=-p_{x}^{*}+\mu_{e} \nabla^{2} u-\frac{\mu}{k} u-\frac{\mu_{m}}{2}\left[H^{2}\right]_{x} \\
\rho\left\{\frac{1}{2}\left[u^{2}+v^{2}\right]_{y}+u\left(v_{x}-u_{y}\right)\right\}=-p_{y}^{*}+\mu_{e} \nabla^{2} v-\frac{\mu}{k} v-\frac{\mu_{m}}{2}\left[H^{2}\right]_{y} \\
u H_{x}+v H_{y}-\frac{1}{\mu_{m} \sigma} \nabla^{2} H=0
\end{gathered}
$$

where $\nabla^{2} \equiv \partial_{x x}+\partial_{y y}$ is the Laplacian in two dimensions, and

$$
p^{*}=p+\frac{\mu_{m}}{2} H^{2}
$$


Equations (9), (10), (11), (12) and (13) represent five scalar equations in the five unknowns $u, v, H, p$ and $p^{*}$ (the magnetic pressure function). These equations govern the two-dimensional flow of a finitely conducting fluid ( $\sigma$ is finite) in a constant permeability porous material in the presence of a magnetic field.

If the fluid is infinitely conducting then $\sigma \rightarrow \infty, \mu_{m} \sigma \rightarrow \infty$ and $\frac{1}{\mu_{m} \sigma} \rightarrow 0$. Diffusion Equation (12) thus reduces to:

$$
u H_{x}+v H_{y}=0
$$

\section{Vorticity-Streamfunction and Generalized Pressure Formulation}

For three dimensional flow, the vorticity vector is the curl of the velocity vector. This definition reduces to the following expression for vorticity, $\omega$, of a fluid in two space dimensions

$$
\omega=v_{x}-u_{y}
$$

Defining the generalized pressure function

$$
h=p^{*}+\frac{1}{2} \rho\left(u^{2}+v^{2}\right)
$$

then Equations (10) and (11) are replaced, respectively, by

$$
\begin{aligned}
& h_{x}-\rho v \omega=\mu_{e} \nabla^{2} u-\frac{\mu}{k} u \\
& h_{y}+\rho u \omega=\mu_{e} \nabla^{2} v-\frac{\mu}{k} v
\end{aligned}
$$

Now, equation of continuity (9) implies the existence of a streamfunction $\psi(x, y)$ such that

$$
u=\psi_{y}
$$

and

$$
v=-\psi_{x}
$$

With the introduction of the streamfunction, the equation of continuity (9) is automatically satisfied, and the vorticity definition (15) becomes

$$
\omega=-\nabla^{2} \psi
$$

Using (19) and (20) in (17), (18), (12) and (14), we obtain, respectively

$$
\begin{gathered}
h_{x}-\rho\left(\psi_{x}\right) \nabla^{2} \psi=\mu_{e} \nabla^{2}\left(\psi_{y}\right)-\frac{\mu}{k}\left(\psi_{y}\right) \\
h_{y}-\rho\left(\psi_{y}\right) \nabla^{2} \psi=-\mu_{e} \nabla^{2}\left(\psi_{x}\right)+\frac{\mu}{k}\left(\psi_{x}\right) \\
\psi_{y} H_{x}-\psi_{x} H_{y}-\frac{1}{\mu_{m} \sigma} \nabla^{2} H=0 \\
\psi_{y} H_{x}-\psi_{x} H_{y}=0
\end{gathered}
$$


Once Equations (21), (22), (23) and (24), or (25), are solved for $\psi, \omega, h$ and $H$ as functions of $x$ and $y$, velocity components can be computed from (19) and (20), then $p^{*}$ can be obtained from (16) and finally $p$ from (13).

\section{Compatibility Equation and the Form of Streamfunction}

The generalized pressure function $h(x, y)$ satisfying (22) and (23) is assumed to be a continuous function with the integrability condition, $h_{x y}=h_{y x}$. Integrability condition leads us to the following compatibility equation, derived by differentiating (22) with respect to $y$ and (23) with respect to $x$, and equating the resulting derivatives:

$$
\rho\left[\left(\psi_{x}\right) \nabla^{2} \psi_{y}-\left(\psi_{y}\right) \nabla^{2} \psi_{x}\right]+\mu_{e} \nabla^{4} \psi=\frac{\mu}{k} \nabla^{2} \psi
$$

or

$$
\rho \frac{\partial\left(\psi, \nabla^{2} \psi\right)}{\partial(x, y)}+\mu_{e} \nabla^{4} \psi=\frac{\mu}{k} \nabla^{2} \psi
$$

where $\nabla^{4} \equiv \partial_{x x x x}+2 \partial_{x x y y}+\partial_{y y y y}$.

The streamfunction $\psi(x, y)$ is a solution to (26) or (27). Its determination is a formidable task at present. We therefore assume that the streamfunction is linear in one of the space dimensions. We thus let

$$
\psi(x, y)=y f(x)+g(x)
$$

Using (28) in (26), and equating coefficients of similar powers of $y$, we obtain the following ordinary differential equations for $f(x)$ and $g(x)$, wherein prime notation denotes ordinary differentiation

$$
\begin{aligned}
& \mu_{e} f^{i v}+\rho\left[f^{\prime \prime} f^{\prime \prime}-f f^{\prime \prime \prime}\right]-\frac{\mu}{k} f^{\prime \prime}=0 \\
& \mu_{e} g^{i v}+\rho\left[g^{\prime} f^{\prime \prime}-f g^{\prime \prime \prime}\right]-\frac{\mu}{k} g^{\prime \prime}=0
\end{aligned}
$$

Integrating (29) and (30) once with respect to $x$, we obtain, respectively:

$$
\begin{aligned}
& \mu_{e} f^{\prime \prime \prime}+\rho\left[\left(f^{\prime}\right)^{2}-f f^{\prime \prime}\right]-\frac{\mu}{k} f^{\prime}=C_{1} \\
& \mu_{e} g^{\prime \prime \prime}+\rho\left[f^{\prime} g^{\prime}-f g^{\prime \prime}\right]-\frac{\mu}{k} g^{\prime}=C_{2}
\end{aligned}
$$

where $C_{1}$ and $C_{2}$ are arbitrary constants, whose values will be discussed following solution of pressure function, below. Once Equations (31) and (32) are solved for $f(x)$ and $g(x)$, the streamfunction given by (28) can then be obtained.

Using (28) in (19) and (20), we obtain the following expressions for the velocity components:

$$
u=\psi_{y}=f
$$

and 


$$
v=-\psi_{x}=-y f^{\prime}-g^{\prime}
$$

Equations (21) and (28) give the following expression for vorticity:

$$
\omega=-y f^{\prime \prime}-g^{\prime \prime}
$$

Using (28) in (22) and (23) we obtain, respectively;

$$
\begin{gathered}
h_{x}=\rho\left(y f^{\prime}+g^{\prime}\right)\left[y f^{\prime \prime}+g^{\prime \prime}\right]+\mu_{e} f^{\prime \prime}-\frac{\mu}{k} f \\
h_{y}=\rho\left(y f f^{\prime \prime}+f g^{\prime \prime}\right)-\mu_{e}\left[y f^{\prime \prime \prime}+g^{\prime \prime \prime}\right]+\left(\frac{\mu}{k} y f^{\prime}+\frac{\mu}{k} g^{\prime}\right)
\end{gathered}
$$

From (31) and (32), we can write

$$
\begin{gathered}
\mu_{e} f^{\prime \prime \prime}-\frac{\mu}{k} f^{\prime}=C_{1}+\rho\left[f f^{\prime \prime}-\left(f^{\prime}\right)^{2}\right] \\
\mu_{e} g^{\prime \prime \prime}-\frac{\mu}{k} g^{\prime}=C_{2}+\rho\left[f g^{\prime \prime}-f^{\prime} g^{\prime}\right]
\end{gathered}
$$

Using (38) and (39) in (36) and (37), followed by integrating (36) and (37), we obtain the following expression for the generalized pressure function $h(x, y)$

$$
h=-\frac{y^{2}}{2}\left[C_{1}-\rho\left(f^{\prime}\right)^{2}\right]-y\left[C_{2}-\rho f^{\prime} g^{\prime}\right]+\frac{\rho}{2}\left(g^{\prime}\right)^{2}+\mu_{e} f^{\prime}-\frac{\mu}{k} \int f \mathrm{~d} x+C_{3}
$$

where $C_{3}$ is an arbitrary constant.

Using (28) in the diffusion Equation (24), we obtain

$$
f H_{x}-\left(y f^{\prime}+g^{\prime}\right) H_{y}-\frac{1}{\mu_{m} \sigma} \nabla^{2} H=0
$$

If the fluid is infinitely conducting, then (40) reduces to

$$
f H_{x}-\left(y f^{\prime}+g^{\prime}\right) H_{y}=0
$$

Using (33), (34) and (40) in (16), we obtain

$$
p^{*}=-C_{1} \frac{y^{2}}{2}-C_{2} y+\mu_{e} f^{\prime}-\frac{1}{2} \rho f^{2}-\frac{\mu}{k} \int f \mathrm{~d} x+C_{3}
$$

while using (43) in (13) gives

$$
p=\left\{-C_{1} \frac{y^{2}}{2}-C_{2} y+\mu_{e} f^{\prime}-\frac{1}{2} \rho f^{2}-\frac{\mu}{k} \int f \mathrm{~d} x+C_{3}\right\}-\frac{\mu_{m}}{2} H^{2}
$$

\section{Determination of the Streamfunction}

In the absence of a general solution to Equations (31) and (32), the functions $f(x)$ and $g(x)$ have customarily be determined in accordance with the following procedure that received attention in the study of Navier-Stokes flow and in MHD flow [1]:

1) Find a particular solution for $f(x)$ satisfying (31).

2) Substitute the solution for $f(x)$, found in step 1), in (32) and find a general solution for $g(x)$.

3) Once $f(x)$ and $g(x)$ are found, the streamfunction $\psi(x, y)$ can be 
calculated using (28).

This procedure has some drawbacks that have been discussed in details in [14] and [15], and include the difficulty of finding particular solutions to (31) for general flows, and the presence of many arbitrary constants in the function $g(x)$. These and other drawbacks can be overcome with the following modified, inverse procedure.

1) Assume the form of the function $g(x)$.

2) Substitute the assumed form of $g(x)$ in (32). This produces an ordinary differential equation for $f(x)$.

3) Solve the equation that arises in step 2) for $f(x)$. Solution obtained in this step will involve some arbitrary constants and the solution must satisfy (31).

4) Substitute $f(x)$ that is obtained in step 3) in (31) and determine the arbitrary constants.

We apply the modified algorithm to obtain a solution to Equations (31) and (32). In order to parallel solutions obtained for flow through porous media without magnetic effects, discussed in [15], we assume the following form of $g(x)$ :

$$
g(x)=\gamma+\alpha \mathrm{e}^{\beta x}
$$

wherein $\alpha, \beta$ and $\gamma$ are known parameters.

Using (45) in (31) and (32) we obtain the following first order ordinary differential equation for $f(x)$ :

$$
f^{\prime}-\beta f=\frac{\mu}{\rho k}-\frac{\mu_{e}}{\rho} \beta^{2}+\frac{C_{2}}{\alpha \beta \rho}
$$

whose solution takes the form

$$
f(x)=\frac{1}{\rho}\left[\mu_{e} \beta-\frac{\mu}{k \beta}-\frac{C_{2}}{\alpha \beta^{2}}\right]+C_{4} \mathrm{e}^{\beta x}
$$

where $C_{4}$ is an arbitrary constant.

Using (45) and (47) in (28), we obtain the following expression for the streamfunction:

$$
\psi(x, y)=\gamma+\frac{1}{\rho}\left[\mu_{e} \beta-\frac{\mu}{k \beta}-\frac{C_{2}}{\alpha \beta^{2}}\right] y+\left(C_{4} y+\alpha\right) \mathrm{e}^{\beta x}
$$

Using (48), velocity components $u$ and $v$, given by Equations (33) and (34), respectively, and vorticity, given by Equation (35), take the following forms, respectively

$$
\begin{gathered}
u=\frac{1}{\rho}\left[\mu_{e} \beta-\frac{\mu}{k \beta}-\frac{C_{2}}{\alpha \beta^{2}}\right]+C_{4} \mathrm{e}^{\beta x} \\
v=-\beta\left[C_{4} y+\alpha\right] \mathrm{e}^{\beta x} \\
\omega=-\beta^{2}\left[C_{4} y+\alpha\right] \mathrm{e}^{\beta x}=\beta \nu
\end{gathered}
$$

The generalized pressure function $h(x, y)$ given by Equation (40), and the pressures $p^{*}$ and $p$, given by Equations (43) and (44), respectively, take the following forms, respectively 


$$
\begin{aligned}
h= & -C_{1} \frac{y^{2}}{2}-C_{2} y+\rho \beta^{2} \mathrm{e}^{2 \beta x}\left(C_{4} y+\alpha\right)^{2}+C_{4} \mathrm{e}^{\beta x}\left(\mu_{e} \beta-\frac{\mu}{\beta k}\right) \\
& -\frac{\mu}{\rho k}\left[\mu_{e} \beta-\frac{\mu}{k \beta}-\frac{C_{2}}{\alpha \beta^{2}}\right] x+C_{5}
\end{aligned}
$$

where $C_{5}$ is an arbitrary constant.

$$
\begin{aligned}
p^{*}= & -C_{1} \frac{y^{2}}{2}-C_{2} y+C_{2} \frac{C_{4}}{\alpha \beta^{2}} \mathrm{e}^{\beta x}-\frac{\rho}{2} C_{4}^{2} \mathrm{e}^{2 \beta x}-\frac{\mu}{\rho k}\left[\mu_{e} \beta-\frac{\mu}{k \beta}-\frac{C_{2}}{\alpha \beta^{2}}\right] x \\
& -\frac{1}{2 \rho}\left(\mu_{e} \beta-\frac{\rho \mu}{2 k \beta}-\frac{C_{2}}{2 \alpha \beta^{2}}\right)^{2}+C_{5} \\
p= & -C_{1} \frac{y^{2}}{2}-C_{2} y+C_{2} \frac{C_{4}}{\alpha \beta^{2}} \mathrm{e}^{\beta x}-\frac{\rho}{2} C_{4}^{2} \mathrm{e}^{2 \beta x}-\frac{\mu}{\rho k}\left[\mu_{e} \beta-\frac{\mu}{k \beta}-\frac{C_{2}}{\alpha \beta^{2}}\right] x \\
& -\frac{1}{2 \rho}\left(\mu_{e} \beta-\frac{\rho \mu}{2 k \beta}-\frac{C_{2}}{2 \alpha \beta^{2}}\right)^{2}+C_{5}-\frac{\mu_{m}}{2} H^{2}
\end{aligned}
$$

Equation (54) requires the determination of $H(x, y)$ in order to determine the pressure. To accomplish this, we consider the cases of infinitely conducting fluid and finitely conducting fluid, discussed in what follows.

If the fluid is infinitely conducting then the diffusion equation is given by Equation (25), or, equivalently, (42). In this case if $H(x, y)=$ constant or if $H(x, y)=R(\psi)$, where $R(\psi)$ is an arbitrary function of $\psi$, then (25) is satisfied. For example, the following function satisfies (42):

$$
\begin{aligned}
H(x, y) & =R(\psi)=\psi=y f(x)+g(x) \\
& =\gamma+\left[\frac{\mu_{e}}{\rho} \beta-\frac{\mu}{\rho k \beta}-\frac{C_{2}}{\rho \alpha \beta^{2}}\right] y+\left(C_{4} y+\alpha\right) \mathrm{e}^{\beta x}
\end{aligned}
$$

For finitely conducting fluid, we follow the procedure introduced by Labropulu [1] which leads us to a solution to the diffusion Equation (41) of the form

$$
H(x, y)=Z(x) F(y)+G(y)
$$

where

$$
Z(x)=\int\left[\mathrm{e}^{\mu_{m} \sigma \int \mathrm{d} x}\right] \mathrm{d} x
$$

and the functions $F(y)$ and $G(y)$ in (56) must be such that

$$
\left(y f^{\prime}+g^{\prime}\right) H_{y}+\frac{1}{\mu_{m} \sigma} H_{y y}=0
$$

or equivalently

$$
\begin{aligned}
& \left\{\left(y f^{\prime}+g^{\prime}\right) F^{\prime}(y)+\frac{1}{\mu_{m} \sigma} F^{\prime \prime}(y)\right\} \int\left[\mathrm{e}^{\mu_{m} \sigma f \mathrm{~d} x}\right] \mathrm{d} x \\
& +\frac{1}{\mu_{m} \sigma} G^{\prime \prime}(y)+\left(y f^{\prime}+g^{\prime}\right) G^{\prime}(y)=0
\end{aligned}
$$

Using (45) and (47), we write (59) as 


$$
\begin{aligned}
& \left\{\left(\beta\left[C_{4} y+\alpha\right] \mathrm{e}^{\beta x}\right) F^{\prime}(y)+\frac{1}{\mu_{m} \sigma} F^{\prime \prime}(y)\right\} \int\left[\mathrm{e}^{\mu_{m} \sigma f f \mathrm{~d} x}\right] \mathrm{d} x+\frac{1}{\mu_{m} \sigma} G^{\prime \prime}(y) \\
& +\left(\beta\left[C_{4} y+\alpha\right] \mathrm{e}^{\beta x}\right) G^{\prime}(y)=0
\end{aligned}
$$

where

$$
Z(x)=\int \exp \left\{\frac{\mu_{m} \sigma}{\rho}\left[\mu_{e} \beta-\frac{\mu}{k \beta}-\frac{C_{2}}{\alpha \beta^{2}}\right] x\right\} \cdot \exp \left\{\mu_{m} \sigma \frac{C_{4}}{\beta} e^{\beta x}\right\} \cdot \exp \left\{\mu_{m} \sigma B\right\} \mathrm{d} x(61)
$$

and $B$ is an arbitrary constant.

For the sake of illustration, if we take $F(y)=0$ in (56), then $H(x, y)=G(y)$, where $G(y)$ satisfies

$$
\left(\beta\left[C_{4} y+\alpha\right] \mathrm{e}^{\beta x}\right) G^{\prime}(y)+\frac{1}{\mu_{m} \sigma} G^{\prime \prime}(y)=0
$$

whose solution is given by

$$
G(y)=\int \exp \left(-\mu_{m} \sigma \beta \mathrm{e}^{\beta x}\left[\frac{C_{4} y^{2}}{2}+\alpha y+C_{6}\right]\right) \mathrm{d} y+C_{7}
$$

where $C_{6}$ and $C_{7}$ are arbitrary constant.

\section{Determination of the Arbitrary Constants}

In the above analysis, we derived expressions for the flow quantities under the assumption that the streamfunction is linear in one of the space variables. Solutions obtained are given by Equations (48) to (54), and (55) or (56), and involve seven arbitrary constants, $C_{1}$ to $C_{7}$.

The expression for $f(x)$ given by Equation (47), namely

$$
f(x)=\frac{1}{\rho}\left[\mu_{e} \beta-\frac{\mu}{k \beta}-\frac{C_{2}}{\alpha \beta^{2}}\right]+C_{4} \mathrm{e}^{\beta x}
$$

contains two arbitrary constants, $C_{2}$ and $C_{4}$. This solution for $f(x)$ must satisfy (29). We can use (29) as a condition to help us determine the arbitrary constants $C_{2}$ and $C_{4}$.

Upon substituting (47) into (29), and simplifying, we obtain the following condition on $C_{2}$ and $C_{4}$

$$
C_{2} C_{4} \frac{\beta}{\alpha}=0
$$

Condition (64) implies that either $C_{2}=0$ or $C_{4}=0$.

Case 1: If $C_{4}=0$ and $C_{2} \neq 0$

When $C_{2} \neq 0$ then the pressure is unbounded, unless the fluid extent is finite. This leads to the following observation.

Observation (1). In the MHD flow at hand, if the fluid is of finite extent then the flow variables are given by

$$
\psi(x, y)=\frac{y}{\rho}\left[\mu_{e} \beta-\frac{\mu}{k \beta}-\frac{C_{2}}{\alpha \beta^{2}}\right]+\gamma+\alpha \mathrm{e}^{\beta x}
$$




$$
\begin{gathered}
u=\frac{1}{\rho}\left[\mu_{e} \beta-\frac{\mu}{k \beta}-\frac{C_{2}}{\alpha \beta^{2}}\right] \\
v=-\alpha \beta \mathrm{e}^{\beta x} \\
\omega=-\alpha \beta^{2} \mathrm{e}^{\beta x}=\beta v \\
h=-C_{1} \frac{y^{2}}{2}-C_{2} y+\rho \alpha^{2} \beta^{2} \mathrm{e}^{2 \beta x}-\frac{\mu}{\rho k}\left[\mu_{e} \beta-\frac{\mu}{k \beta}-\frac{C_{2}}{\alpha \beta^{2}}\right] x+C_{5} \\
p^{*}=-C_{1} \frac{y^{2}}{2}-C_{2} y-\frac{\mu}{\rho k}\left[\mu_{e} \beta-\frac{\mu}{k \beta}-\frac{C_{2}}{\alpha \beta^{2}}\right] x \\
-\frac{1}{2 \rho}\left(\mu_{e} \beta-\frac{\rho \mu}{2 k \beta}-\frac{C_{2}}{2 \alpha \beta^{2}}\right)^{2}+C_{5} \\
p=-C_{1} \frac{y^{2}}{2}-C_{2} y-\frac{\mu}{\rho k}\left[\mu_{e} \beta-\frac{\mu}{k \beta}-\frac{C_{2}}{\alpha \beta^{2}}\right] x \\
-\frac{1}{2 \rho}\left(\mu_{e} \beta-\frac{\rho \mu}{2 k \beta}-\frac{C_{2}}{2 \alpha \beta^{2}}\right)^{2}+C_{5}-\frac{\mu_{m}}{2} H^{2}
\end{gathered}
$$

where

$$
\begin{gathered}
H(x, y)=Z(x) F(y)+G(y) \\
Z(x)=\int \exp \left\{\frac{\mu_{m} \sigma}{\rho}\left[\mu_{e} \beta-\frac{\mu}{k \beta}-\frac{C_{2}}{\alpha \beta^{2}}\right] x\right\} \cdot \exp \left\{\mu_{m} \sigma B\right\} \mathrm{d} x
\end{gathered}
$$

and $F(y)$ and $G(y)$ satisfy

$$
\left\{\alpha \beta \mathrm{e}^{\beta x} F^{\prime}(y)+\frac{1}{\mu_{m} \sigma} F^{\prime \prime}(y)\right\} Z(x)+\frac{1}{\mu_{m} \sigma} G^{\prime \prime}(y)+\alpha \beta \mathrm{e}^{\beta x} G^{\prime}(y)=0
$$

If in the above solutions, $k \rightarrow \infty$, we recover solution to flow through a free-space domain, that is a domain without the presence of a porous matrix.

Case 2: If $C_{2}=C_{4}=0$

For fluid of infinite extend, we take $C_{1}=C_{2}=0$ so that the pressure is bounded. Taking $C_{4}=0$ as well leads to the following observation.

Observation (2). In the MHD flow at hand, if the fluid is of infinite extent and $C_{4}=0$, then the flow variables are given by

$$
\begin{gathered}
\psi(x, y)=\frac{y}{\rho}\left[\mu_{e} \beta-\frac{\mu}{k \beta}\right]+\gamma+\alpha \mathrm{e}^{\beta x} \\
u=\frac{1}{\rho}\left[\mu_{e} \beta-\frac{\mu}{k \beta}\right] \\
v=-\alpha \beta \mathrm{e}^{\beta x} \\
\omega=-\alpha \beta^{2} \mathrm{e}^{\beta x}=\beta v \\
h=\rho \alpha^{2} \beta^{2} \mathrm{e}^{2 \beta x}-\frac{\mu}{\rho k}\left[\mu_{e} \beta-\frac{\mu}{k \beta}\right] x+C_{5} \\
p^{*}=-\frac{\mu}{\rho k}\left[\mu_{e} \beta-\frac{\mu}{k \beta}\right] x-\frac{1}{2 \rho}\left(\mu_{e} \beta-\frac{\rho \mu}{2 k \beta}\right)^{2}+C_{5}
\end{gathered}
$$




$$
p=-\frac{\mu}{\rho k}\left[\mu_{e} \beta-\frac{\mu}{k \beta}\right] x-\frac{1}{2 \rho}\left(\mu_{e} \beta-\frac{\rho \mu}{2 k \beta}\right)^{2}+C_{5}-\frac{\mu_{m}}{2} H^{2}
$$

where

$$
\begin{gathered}
H(x, y)=Z(x) F(y)+G(y) \\
Z(x)=\int \exp \left\{\frac{\mu_{m} \sigma}{\rho}\left[\mu_{e} \beta-\frac{\mu}{k \beta}\right] x\right\} \cdot \exp \left\{\mu_{m} \sigma B\right\} \mathrm{d} x
\end{gathered}
$$

and $F(y)$ and $G(y)$ satisfy

$$
\left\{\alpha \beta \mathrm{e}^{\beta x} F^{\prime}(y)+\frac{1}{\mu_{m} \sigma} F^{\prime \prime}(y)\right\} Z(x)+\frac{1}{\mu_{m} \sigma} G^{\prime \prime}(y)+\alpha \beta \mathrm{e}^{\beta x} G^{\prime}(y)=0
$$

If in the above solutions, $k \rightarrow \infty$, we recover solution to flow through a free-space domain, that is a domain without the presence of a porous matrix.

Case 3: If $C_{4} \neq 0$ and $C_{2}=0$

For fluid of infinite extent, coefficients of $y$ and $y^{2}$ must vanish in order for the pressure function to be bounded. We therefore take $C_{1}=C_{2}=0$ and state the following observation.

Observation (3). In the MHD flow at hand, if the fluid is of infinite extent and $C_{4} \neq 0$, then the flow variables are given by

$$
\begin{aligned}
& \psi(x, y)=\gamma+\frac{1}{\rho}\left[\mu_{e} \beta-\frac{\mu}{k \beta}\right] y+\left(C_{4} y+\alpha\right) \mathrm{e}^{\beta x} \\
& u=1 / \rho\left[\mu_{e} \beta-\frac{\mu}{k \beta}\right]+C_{4} \mathrm{e}^{\beta x} \\
& v=-\beta\left[C_{4} y+\alpha\right] \mathrm{e}^{\beta x} \\
& \omega=-\beta^{2}\left[C_{4} y+\alpha\right] \mathrm{e}^{\beta x}=\beta v \\
& h=\rho \beta^{2} \mathrm{e}^{2 \beta x}\left(C_{4} y+\alpha\right)^{2}+C_{4} \mathrm{e}^{\beta x}\left(\mu_{e} \beta-\frac{\mu}{\beta k}\right)-\frac{\mu}{\rho k}\left[\mu_{e} \beta-\frac{\mu}{k \beta}\right] x+C_{5} \\
& p^{*}=-\frac{\rho}{2} C_{4}^{2} \mathrm{e}^{2 \beta x}-\frac{\mu}{\rho k}\left[\mu_{e} \beta-\frac{\mu}{k \beta}\right] x-\frac{1}{2 \rho}\left(\mu_{e} \beta-\frac{\rho \mu}{2 k \beta}\right)^{2}+C_{5} \\
& p=-\frac{\rho}{2} C_{4}^{2} \mathrm{e}^{2 \beta x}-\frac{\mu}{\rho k}\left[\mu_{e} \beta-\frac{\mu}{k \beta}\right] x-\frac{1}{2 \rho}\left(\mu_{e} \beta-\frac{\rho \mu}{2 k \beta}\right)^{2}+C_{5}-\frac{\mu_{m}}{2} H^{2} \\
& H(x, y)=Z(x) F(y)+G(y) \\
& Z(x)=\int \exp \left\{\frac{\mu_{m} \sigma}{\rho}\left[\mu_{e} \beta-\frac{\mu}{k \beta}\right] x\right\} \cdot \exp \left\{\mu_{m} \sigma \frac{C_{4}}{\beta} \mathrm{e}^{\beta x}\right\} \cdot \exp \left\{\mu_{m} \sigma B\right\} \mathrm{d} x
\end{aligned}
$$

and $B$ is an arbitrary constant, and the functions $F(y)$ and $G(y)$ satisfy

$$
\begin{aligned}
& \left\{\left(\beta\left[C_{4} y+\alpha\right] \mathrm{e}^{\beta x}\right) F^{\prime}(y)+\frac{1}{\mu_{m} \sigma} F^{\prime \prime}(y)\right\} \int\left[\mathrm{e}^{\mu_{m} \sigma f f \mathrm{dx}}\right] \mathrm{d} x \\
& +\frac{1}{\mu_{m} \sigma} G^{\prime \prime}(y)+\left(\beta\left[C_{4} y+\alpha\right] \mathrm{e}^{\beta x}\right) G^{\prime}(y)=0
\end{aligned}
$$


If in the above solutions, $k \rightarrow \infty$, we recover solution to flow through a free-space domain, that is a domain without the presence of a porous matrix.

In all of the cases discussed above, all remaining arbitrary constants must be determined using physical conditions on the flow variables.

\section{Potential Flow}

Using the presented method of solution, if we choose $g(x)$ as a linear function in the variable $x$, namely

$$
g(x)=C x+D
$$

where $C$ and $D$ are known parameters, Equations (31) and (32) yield

$$
f(x)=\frac{1}{\rho}\left(\frac{\mu}{k}+\frac{C_{2}}{C}\right) x+C_{8}
$$

and Equation (28) gives the following expression for the streamfunction:

$$
\psi(x, y)=y\left[\frac{1}{\rho}\left(\frac{\mu}{k}+\frac{C_{2}}{C}\right) x+C_{8}\right]+C x+D
$$

Velocity components take the form:

$$
\begin{array}{r}
u=\frac{1}{\rho}\left(\frac{\mu}{k}+\frac{C_{2}}{C}\right) x+C_{8} \\
v=-\frac{1}{\rho}\left[\frac{\mu}{k}+\frac{C_{2}}{C}\right] y-C
\end{array}
$$

and the vorticity vanishes since

$$
\omega=-\nabla^{2} \psi=-y f^{\prime \prime}-g^{\prime \prime}=0
$$

If in the above solutions, $k \rightarrow \infty$, we recover solution to flow through a free-space domain, that is a domain without the presence of a porous matrix.

\section{Conclusion}

In this work, we considered plane transverse MHD flow through a porous structure of contant permeability. In order to solve the governing equations, we assumed the streamfunction to be linear in one of the space variables, namely $\psi=y f(x)+g(x)$. We derived a compatibility equation that resulted in two coupled ordinary differential equations the solution to which comprises solution for the streamfunction of the flow. In order to avoid the presence of a large number of arbitrary constants, we implemented an inverse approach to obtain the solution. Advantage to this implemented approach is avoidance of Riabouchinsky assumption that $g(x)$ must be taken as zero. We have obtained various forms of the flow variables depending on three observations made about some of the arbitrary constants. Solutions obtained are for both cases of finitely conducting and infinitely conducting fluids, and produce same forms of solutions obtained by Labropulu [1], when permeability goes to infinity. It is also worth noting that solutions for the streamfunction, velocity and viscosity are 
dependent on permeability of the medium and are the same results obtained by Kamel and Hamdan [15].

\section{Acknowledgements}

The authors wish to express their sincere thanks to the referees of this work who constructive suggestions lead to enhancing this work.

\section{Conflicts of Interest}

The authors declare no conflicts of interest regarding the publication of this paper.

\section{References}

[1] Lapropulu, F. (1987) Riabouchinsky Flows in Magnetohydrodynamics. M.Sc Thesis, University of Windsor, Windsor.

[2] Raptis, A. and Perdikis, C. (1985) Magnetohydrodynamics Effects on Mass Transfer Flow through Porous Medium. Astrophysics and Space Science, 113, 53-58. https://doi.org/10.1007/BF00650270

[3] Chandna, O.P. and Labropulu, F. (1992) Riabouchinsky Flows in Magnetohydrodynamics. Quarterly of Applied Mathematics, 50, 273-289. https://doi.org/10.1090/qam/1162276

[4] Geindreau, C. and Auriault, J.-L. (2002) Magnetohydrodynamic Flows in Porous Media. Journal of Fluid Mechanics, 466, 343-363. https://doi.org/10.1017/S0022112002001404

[5] Kaloni, P.N. and Huschilt, K. (1984) Semi-Inverse Solution of a Non-Newtonian Fluid. International Journal Non-Linear Mechanics, 19, 373-381. https://doi.org/10.1016/0020-7462(84)90065-9

[6] Kingston, J. and Power, G. (1968) An Analysis of Two-Dimensional Aligned-Field Magnetohydrodynamic Flows. Journal of Applied Mathematics and Physics, 19, 851-863. https://doi.org/10.1007/BF01602266

[7] Rothmayer, A.P. (2014) Magnetohydrodynamic Channel Flows with Weak Transverse Magnetic Fields. Philosophical Transactions of the Royal Society A, 372, 1-12. https://doi.org/10.1098/rsta.2013.0344

[8] Labropulu, F. and Chandna, O.P. (2000) Exact Solutions of Steady Plane Flows Using $(r, \Psi)$ Coordinates. International Journal of Mathematics and Mathematical Sciences, 23, 449-475. https://doi.org/10.1155/S0161171200001952

[9] Jabeen, K., Mushtaq, M. and Akram, R.M. (2019) A Comparative Study of MHD Flow Analysis in a Porous Medium by Using Differential Transformation Method and Variational Iteration Method. Journal of Contemporary Applied Mathematics, 9, 10-24.

[10] Kuzhir, P., Bossis, G., Bashtovoi, V. and Volkovab, O. (2003) Flow of Magnetorheological Fluid through Porous Media. European Journal of Mechanics B Fluids, 22, 331-343. https://doi.org/10.1016/S0997-7546(03)00040-2

[11] Rudraiah, N. and Kalal, M. (2007) Electrohydrodynamic Surface Instabilities: Role of Porous Lining at the Ablative Surface of Laser-Driven Inertial Fusion Energy Target. Current Science, 93, 628-647.

[12] Chandrasekhara, B.C., Rajani, K. and Rudraiah, N. (1978) Effect of Slip on Porous-Walled Squeeze Films in the Presence of a Magnetic Field. Applied Scientific 
Research, 34, 393-411. https://doi.org/10.1007/BF00383973

[13] Rudraiah, N., Ramaiah, B. and Rajasekhar, B. (1975) Hartmann Flow over a Permeable Bed. International Journal of Engineering Science, 13, 1-24. https://doi.org/10.1016/0020-7225(75)90070-1

[14] Hamdan, M.H. (1998) An Alternative Approach to Exact Solutions of a Special Class of Navier-Stokes Flows. Journal of Applied Mathematics and Computation, 93, 83-90. https://doi.org/10.1016/S0096-3003(97)10047-9

[15] Kamel, M.T. and Hamdan, M.H. (2006) Riabouchinsky Flow through Porous Media. International Journal of Pure and Applied Mathematics, 27, 113-126.

\section{Nomenclature}

$B, C, D, C_{1}, C_{2}, \cdots, C_{8}:$ arbitrary constants

$f, g, Z$ : functions of $x$

$F, G$ : functions of $y$

$R$ : arbitrary function of the streamfunction $\psi$

$\alpha, \beta, \gamma:$ arbitrary parameters

$\mu$ : base fluid viscosity coefficient

$\mu_{e}:$ effective viscosity

$\mu_{m}:$ magnetic permeability

$x, y:$ space variables

$v$ : velocity vector field

$u$ : tangential velocity component

$v$. normal velocity component

$h$ : generalized pressure function

$\boldsymbol{H}$ : magnetic field

$H$ : magnetic field third component

$p$ : pressure function

$p^{*}:$ magnetic pressure function

$\rho$ : fluid density

$k$ : permeability

$\sigma$ : electrical conductivity

$\omega$ : vorticity vector third component

$\psi:$ streamfunction

$\nabla:$ gradient operator

$\nabla^{2}$ : Laplacian operator

$\nabla \times$ : curl operator 